\title{
SOME MORE RESULTS ON RATES OF CONVERGENCE IN THE LAW OF LARGE NUMBERS FOR WEIGHTED SUMS OF INDEPENDENT RANDOM VARIABLES( $\left.{ }^{1}\right)$
}

\author{
BY \\ D. L. HANSON AND F. T. WRIGHT( $\left.{ }^{2}\right)$
}

1. Introduction and summary. Let $X_{N}$ for $N=1,2, \ldots$ be an independent sequence of random variables with finite first absolute moments; let $a_{N, k}$ for $N, k=1,2, \ldots$ be real numbers; let

$$
S_{N}=\sum_{k=1}^{\infty} a_{N, k}\left(X_{k}-E X_{k}\right)
$$

and let

$$
A_{N}=\frac{1}{N} \sum_{k=1}^{N}\left(X_{k}-E X_{k}\right) .
$$

Both classical and modern results deal with the convergence of the sequences $A_{N}$ and $P\left\{\left|A_{N}\right|>\varepsilon\right\}$ to zero, this convergence being of obvious practical interest. In the past few years sums of the form (1.1) have received attention, partly due to the natural tendency of mathematicians to generalize known results, and partly because sums of the form

$$
\frac{1}{N} \sum_{k=1}^{N}\left[\sum_{j=0}^{\infty} a_{j}\left(X_{k-j}-E X_{k-j}\right)\right]
$$

are of interest in certain practical statistical and probabilistic problems. We have not attempted to provide a complete listing of the work done in this area. [1] through [11] contain several different types of current work, and provide a fairly large, though by no means complete, bibliography for this area.

The results given here extend and sharpen the results of [6] giving rates of convergence of $P\left\{\left|S_{N}\right|>\varepsilon\right\}$ to zero. There are five theorems in [6], each involving as a hypothesis some condition closely related to the existence of $E\left|X_{k}-E X_{k}\right|^{t}$. In the next section we extend and sharpen some of these theorems by considering separately the cases $t \leqq 2$ and $t>2$; in addition, we provide a theorem giving series convergence under weaker moment conditions than were used in [6] but under

Received by the editors June 23, 1968.

(') Research partially sponsored by the Air Force Office of Scientific Research, Office of Aerospace Research, United States Air Force, under Grant No. AF-AFOSR-746-65 and under Grant No. AFOSR-68-1394.

$\left.{ }^{2}\right)$ Part of this research was done while this author held an NDEA Graduate Fellowship at the University of Missouri at Columbia. 
stronger assumptions on the $a_{N, k}$ 's. In $\S 3$ we show that Theorem 5 of [6] is actually a corollary to Theorem 4 of [6] and investigate the sharpness of Theorem 4 . $\S 4$ contains some miscellaneous concluding remarks.

2. Extensions of previous results. Throughout this paper $C$ will denote various positive constants whose exact numerical values do not matter. Using this notation inequalities such as $1+C \leqq C$ are valid.

Let $X_{N}, a_{N, \dot{k}}$, and $S_{N}$ be defined as in $\S 1$. Suppose $t$ and $t^{\prime}$ are constants and $\left\{\alpha_{N}\right\},\left\{\gamma_{N}\right\}$, and $\left\{\rho_{N}\right\}$ are sequences of positive numbers such that

$$
\begin{gathered}
\sum_{k}\left|a_{N, k}\right|^{t^{\prime}} \leqq \alpha_{N}, \\
\sum_{k} a_{N, k}^{2} \leqq \gamma_{N},
\end{gathered}
$$

and

$$
\sum_{k}\left|a_{N, k}\right|^{t} \leqq \rho_{N}
$$

For $y \geqq 0$ define

$$
F(y)=\sup _{k} P\left\{\left|X_{k}-E X_{k}\right| \geqq y\right\} .
$$

We will prove the following theorems:

THEOREM 1a. If $1 \leqq t \leqq 2$ and $E\left|X_{k}-E X_{k}\right|^{t} \leqq M<\infty$ for all $k$, then for every $\varepsilon>0$

$$
P\left\{\left|S_{N}\right|>\varepsilon\right\} \leqq O\left(\rho_{N}\right) .
$$

THEOREM 1b. If $1 \leqq t^{\prime}<t \leqq 2$, if $y^{t} F(y) \leqq M<\infty$ for all $y>0$, and if there exists a $\lambda_{0}$ such that $\alpha_{N} \rho_{N}^{\lambda_{0}}<M^{\prime}<\infty$ for all $N$, then (2.5) holds for every $\varepsilon>0$.

THEOREM 1c. If $t>2$, if $y^{t} F(y) \leqq M<\infty$ for all $y>0$, and if there exists $a \nu_{0}>0$ such that $\gamma_{N}^{v_{0}} \leqq O\left(\rho_{N}\right)$, then (2.5) holds for every $\varepsilon>0$.

THEOREM 2a. If $1 \leqq t \leqq 2$, if $E\left|X_{k}-E X_{k}\right|^{t} \leqq M<\infty$ for all $k$, if $y^{t} F(y) \rightarrow 0$ as $y \rightarrow \infty$, and if $\rho_{N} \rightarrow 0$ as $N \rightarrow \infty$, then for every $\varepsilon>0$

$$
P\left\{\left|S_{N}\right|>\varepsilon\right\}=o\left(\rho_{N}\right) .
$$

THEOREM 2b. If $1 \leqq t^{\prime}<t \leqq 2$, if $y^{t} F(y) \rightarrow 0$ as $y \rightarrow \infty$, if $\rho_{N} \rightarrow 0$ as $N \rightarrow \infty$, and if there exists a $\lambda_{0}$ such that $\alpha_{N} \rho_{N}^{\lambda_{0}} \leqq M<\infty$ for all $N$, then (2.6) holds for every $\varepsilon>0$.

THEOREM 2c. If $t>2$, if $y^{t} F(y) \rightarrow 0$ as $y \rightarrow \infty$, if $\rho_{N} \rightarrow 0$ as $N \rightarrow \infty$, and if there exists a $\nu_{0}>0$ such that $\gamma_{N}^{\nu_{0}} \leqq O\left(\rho_{N}\right)$, then (2.6) holds for every $\varepsilon>0$.

In Theorems 3, 4, and 5 we assume that $\alpha_{N}, \gamma_{N}$, and $\rho_{N}$ are of the form $C N^{\alpha}$, $C N^{-\gamma}$, and $C N^{-\rho}$ respectively. In addition, for Theorems 3 and 5 we assume there exist constants $C$ and $\beta$ such that

$$
\max _{k}\left|a_{N, k}\right| \leqq C N^{-\beta} .
$$


For $s=t$ and $s=2$,

$$
\left[\max _{k}\left|a_{N, k}\right|\right]^{s} \leqq \sum_{k}\left|a_{N, k}\right|^{s}
$$

so we may assume

$$
\beta t \geqq \rho \quad \text { and } \quad 2 \beta \geqq \gamma
$$

For $t>2$

$$
\sum_{k}\left|a_{N, k}\right|^{t} \leqq\left[\max _{k}\left|a_{N, k}\right|\right]^{t-2} \sum_{k} a_{N, k}^{2}
$$

so if $t>2$ we may assume

$$
\rho \geqq \beta(t-2)+\gamma
$$

Define for $N, M=1,2, \ldots$

$$
\nu_{N, M}=\text { cardinality }\left\{k:\left|a_{N, k}\right| \geqq M^{-1}\right\} .
$$

THEOREM 3. If $t>2, \gamma>0$, and $F$ satisfies

$$
\lim _{y \rightarrow \infty} F(y)=0 \text { and } \int_{0}^{\infty} y^{t}|d F(y)|<\infty,
$$

then for every $\varepsilon>0$

$$
\sum_{N} N^{\beta(t-2)+\gamma-1} P\left\{\left|S_{N}\right|>\varepsilon\right\}<\infty .
$$

THEOREM 4. If $t \geqq 1 ; \rho>0 ; \gamma>0$; and there exists a nonnegative and nonincreasing real valued function $G$ satisfying (2.11), $G(x) \geqq F(x)$ for all $x$, and

$$
\sup _{x \geqq 1} \sup _{y \geqq x} \frac{y^{t} F(y)}{x^{t} G(x)}=\eta<\infty,
$$

then for every $\varepsilon>0$

$$
\sum_{N} N^{\rho-1} P\left\{\left|S_{N}\right|>\varepsilon\right\}<\infty
$$

THEOREM 5. If $t \geqq 1, \rho>0, \gamma>0, F$ satisfies (2.11), and either

(a) $\nu_{N, M} \leqq C M^{t} N^{-(\rho-1)} g(N)$ with $\sum_{N} g(N)<\infty$, or

(b) $\nu_{N, M} \leqq C M^{\mu} N^{-\sigma}$ with $\sigma \geqq \mu \beta$, and either $\rho \neq \sigma$ or $t \neq \mu$, then (2.14) holds for every $\varepsilon>0$.

Proofs. Throughout these proofs summations will be taken over those values of $k$ for which $a_{N, k} \neq 0$. Initially we prove Theorems $1 \mathrm{a}, 1 \mathrm{~b}$, and $1 \mathrm{c}$ under the assumption that $\rho_{N} \rightarrow 0$ as $N \rightarrow \infty$; the other cases will be taken care of at the end of this section. 
The proofs begin as those in [6] where the following inequality was proved:

$$
\begin{aligned}
P\left\{\left|S_{N}\right|>\right. & 3 \varepsilon\} \\
\leqq & \sum_{k} P\left\{\left|a_{N, k}\left(X_{k}-E X_{k}\right)\right|>\varepsilon\right\} \\
& +\sum_{j \neq k} P\left\{\left|a_{N, k}\left(X_{k}-E X_{k}\right)\right|>\delta_{N}\right\} P\left\{\left|a_{N, j}\left(X_{j}-E X_{j}\right)\right|>\delta_{N}\right\} \\
& +P\left\{\left|\sum_{k} a_{N, k} E Y_{N, k}\right|>\varepsilon\right\} \\
& +P\left\{\left|\sum_{k} a_{N, k}\left(Y_{N, k}-E Y_{N, k}\right)\right|>\varepsilon\right\},
\end{aligned}
$$

where $\delta_{N}$ is a sequence of positive numbers to be chosen later and

$$
\begin{aligned}
Y_{N, k} & =X_{k}-E X_{k} & & \text { if }\left|a_{N, k}\left(X_{k}-E X_{k}\right)\right| \leqq \delta_{N} \\
& =0 & & \text { otherwise. }
\end{aligned}
$$

The proof of each theorem proceeds by showing that expressions (2.15)-(2.18) tend to zero at the rate specified in the theorem.

EXPRESSION (2.15). For Theorems 1a, 1b, 1c, 2a, 2b, and 2c, we observe that (2.15) is bounded by

$$
\sum_{k} \frac{\left|a_{N, k}\right|^{t}}{\varepsilon^{t}} \sup _{y \geqq \varepsilon /\left|a_{N, k}\right|}\left[y^{t} P\left\{\left|X_{k}-E X_{k}\right| \geqq y\right\}\right] \leqq C \rho_{N} \sup _{y^{t} \geqq C \rho_{N}^{-1}}\left[y^{t} F(y)\right] .
$$

The hypotheses of Theorems $1 \mathrm{a}, 1 \mathrm{~b}$, and $1 \mathrm{c}$ insure that

$$
\sup _{k} \sup _{y \geqq \delta /\left|a_{N}, k\right|} y^{t} P\left\{\left|X_{k}-E X_{k}\right| \geqq y\right\}<\infty
$$

so that (2.15) is $O\left(\rho_{N}\right)$. The hypotheses of Theorems $2 \mathrm{a}, 2 \mathrm{~b}$, and $2 \mathrm{c}$ insure that

$$
\sup _{y^{t} \geqq C \rho_{N}^{-1}}\left[y^{t} F(y)\right] \rightarrow 0 \quad \text { as } N \rightarrow \infty .
$$

Hence (2.15) is $o\left(\rho_{N}\right)$ under the hypotheses of these theorems.

For Theorem 3 we note that

$$
\begin{array}{rl}
\sum_{N} N^{\beta(t-2)+\gamma-1} \sum_{k} & P\left\{\left|a_{N, k}\left(X_{k}-E X_{k}\right)\right|>\varepsilon\right\} \\
& \leqq \sum_{N} N^{\beta(t-2)+\gamma-1} \sum_{k} F\left(\varepsilon /\left|a_{N, k}\right|\right) \\
& \leqq \sum_{M=1}^{\infty} F(M-1) \sum_{\left\{(N, k): M-1<\varepsilon \| \mid a_{N, k} \leqq M\right\}} N^{\beta(t-2)+\gamma-1} \\
& =\sum_{M=1}^{\infty}[F(M-1)-F(M)] \sum_{N=1}^{\infty} N^{\beta(t-2)+\gamma-1} \nu_{N, M}(\varepsilon),
\end{array}
$$

where $\nu_{N, M}(\varepsilon)=$ card $\left\{k: \varepsilon /\left|a_{N, k}\right| \leqq M\right\}$. Since $\left(\varepsilon^{2} / M^{2}\right) \nu_{N, M}(\varepsilon) \leqq C N^{-\gamma}$ and $\left|a_{N, k}\right| \leqq$ $C N^{-\beta}$ for all $k$, it is clear that $\nu_{N, M}(\varepsilon) \leqq C M^{2} N^{-\gamma}$ and $\nu_{N, M}(\varepsilon)=0$ unless $N \leqq C M^{1 / \beta}$. 
Thus (2.19) is. bounded by

$$
\begin{aligned}
C \sum_{M=1}^{\infty}[F(M-1)- & F(M)] M^{2} \sum_{N=1}^{\left[C M^{1 / \beta}\right]} N^{\beta(t-2)-1} \\
& \leqq C \sum_{M=1}^{\infty}[F(M-1)-F(M)] M^{t} \leqq C+C \int_{0}^{\infty} x^{t}|d F(x)|<\infty .
\end{aligned}
$$

Under the assumptions of Theorem 4 the finiteness of

$$
\sum_{N} N^{\rho-1} \sum_{k} P\left\{\left|a_{N, k}\left(X_{k}-E X_{k}\right)\right|>\varepsilon\right\}
$$

is proved on pages 351 and 352 of [6] starting at (20).

An argument similar to that given for Theorem 3 shows that (2.20) is bounded by

$$
C \sum_{M=1}^{\infty}[F(M-1)-F(M)] \sum_{N=1}^{\left[C M^{1 / \beta]}\right.} N^{\rho-1} \nu_{N, M}(\varepsilon) .
$$

It is clear that there exists a positive integer $r$ such that $\nu_{N, M}(\varepsilon) \leqq \nu_{N, r M}$, and so under assumption (a) of Theorem 5 expression (2.21) is bounded by

$$
C \sum_{M=1}^{\infty}[F(M-1)-F(M)] M^{t} \sum_{N=1}^{\left[C M^{1 / \beta_{]}}\right.} g(N) \leqq C+C \int_{0}^{\infty} x^{t}|d F(x)|<\infty .
$$

Under assumption (b) of Theorem 5 expression (2.21) is bounded by

$$
C \sum_{M=1}^{\infty}[F(M-1)-F(M)] M^{\mu} \sum_{N=1}^{\left[C M^{1 / \beta]}\right.} N^{\rho-1-\sigma} .
$$

If $\rho \neq \sigma$ and $\sigma \geqq \mu \beta$, then $\sum_{N=1}^{\left[C M^{1 / \beta}\right]} N^{\rho-1-\sigma} \leqq C M^{(\rho-\sigma) / \beta}$; from (2.8) we get

$$
\frac{\rho-(\sigma-\beta \mu)}{\beta} \leqq \frac{\rho}{\beta} \leqq t ;
$$

thus expression (2.22) is bounded by

$$
C \sum_{M=1}^{\infty}[F(M-1)-F(M)] M^{t} \leqq C+C \int_{0}^{\infty} x^{t}|d F(x)|<\infty .
$$

If $\rho=\sigma$, then by hypothesis $\mu \neq t$ and this implies that $\mu<t$. Thus (2.22) is bounded by

$$
\begin{aligned}
C \sum_{M=1}^{\infty}[F(M-1)-F(M)] M^{\mu} \sum_{N=1}^{\left[C M^{1 / \beta}\right]} N^{-1} \\
\quad \leqq C \sum_{M=1}^{\infty}[F(M-1)-F(M)] M^{\mu} \log (M+1) \\
\leqq C \sum_{M=1}^{\infty}[F(M-1)-F(M)] M^{t} \leqq C+C \int_{0}^{\infty} x^{t}|d F(x)|<\infty .
\end{aligned}
$$


EXPRESSION (2.16). Expression (2.16) is bounded by

$$
\left[\sum_{k}\left|a_{N, k}\right|^{t} \delta_{N}^{-t}\left(\left(\frac{\delta_{N}}{\left|a_{N, k}\right|}\right)^{t} P\left\{\left|X_{k}-E X_{k}\right|>\frac{\delta_{N}}{\left|a_{N, k}\right|}\right\}\right)\right]^{2} .
$$

The hypotheses of all the theorems insure that $y^{t} F(y) \leqq M<\infty$ for all $y>0$, so that (2.16) is bounded by $C \rho_{N}^{2} \delta_{N}^{-2 t}$. In order for (2.16) to tend to zero at the appropriate rates it would suffice to show that there exists a $\tau>0$ such that

$$
\rho_{N}^{1-\tau} \delta_{N}^{-2 t} \rightarrow 0 \text { as } N \rightarrow \infty .
$$

The $\delta_{N}$ 's will be chosen later in the proof and will satisfy (2.23).

EXPRESSION (2.17). For all the theorems in question it suffices to show that

$$
\sum_{k} a_{N, k} E Y_{N, k} \rightarrow 0 \text { as } N \rightarrow \infty .
$$

For Theorems 1a, 2a, 3, 4, and 5 we observe that

$$
\begin{aligned}
\left|\sum_{k} a_{N, k} E Y_{N, k}\right| & \leqq\left.\sum_{k}\left|a_{N, k}\right|\right|_{\left(\left|a_{N, k}\left(X_{k}-E X_{k}\right)\right| \leqq \delta_{N}\right)}\left(X_{k}-E X_{k}\right) d P \mid \\
& =\sum_{k}\left|a_{N, k}\right| \int_{\left(\left|a_{N, k}\left(X_{k}-E X_{k}\right)\right|>\delta_{N}\right)}\left(X_{k}-E X_{k}\right) d P \mid \\
& \leqq \sum_{k}\left|a_{N . k}\right| \int_{\left(\left|a_{N, k}\left(X_{k}-E X_{k}\right)\right|>\delta_{N}\right)}\left|X_{k}-E X_{k}\right| d P \\
& \leqq \delta_{N}^{-(t-1)} \sum_{k}\left|a_{N, k}\right|^{t} E\left|X_{k}-E X_{k}\right|^{t} \leqq C \delta_{N}^{(t-1)} \rho_{N} .
\end{aligned}
$$

So Expression (2.17) tends to zero at the rates specified if

$$
\delta_{N}^{-(t-1)} \rho_{N} \rightarrow 0 \text { as } N \rightarrow \infty .
$$

We will choose $\left\{\delta_{N}\right\}$ later so as to satisfy (2.24).

For Theorems $1 b, 1 \mathrm{c}, 2 \mathrm{~b}$, and $2 \mathrm{c}$ an argument similar to the above gives

$$
\left|\sum_{k} a_{N, k} E Y_{N, k}\right| \leqq \delta_{N}^{-(t-\lambda-1)} \sum_{k}\left|a_{N, k}\right|^{t-\lambda} E\left|X_{k}-E X_{k}\right|^{t-\lambda}
$$

where $\lambda$ satisfies $0<\lambda<t-1$ and will be chosen later. The hypotheses of the theorems under consideration insure $y^{t} F(y) \leqq M<\infty$ for all $y>0$, which implies that for a fixed $\lambda$ satisfying $0<\lambda<t-1$ there exists an $M^{\prime}$ with

$$
E\left|X_{k}-E X_{k}\right|^{t-\lambda} \leqq M^{\prime}<\infty \text { for all } k \text {. }
$$

If $t>2$, by applying Hölder's inequality and requiring

$$
0<\lambda<t-2 \text {, }
$$

we see that 


$$
\begin{aligned}
\sum_{k}\left|a_{N, k}\right|^{t-\lambda} & =\sum_{k}\left|a_{N, k}\right|^{2 \lambda /(t-2)}\left|a_{N, k}\right|^{t(t-2-\lambda) /(t-2)} \\
& \leqq\left(\sum_{k} a_{N, k}^{2}\right)^{\lambda /(t-2)}\left(\sum_{k}\left|a_{N, k}\right|^{t}\right)^{(t-2-\lambda) /(t-2)} \\
& \leqq \gamma_{N}^{\lambda(t-2)} \rho_{N}^{1-\lambda /(t-2)}
\end{aligned}
$$

so for Theorems $1 \mathrm{c}$ and $2 \mathrm{c}$ it suffices to show that

$$
\delta_{N}^{-(t-\lambda-1)} \gamma_{N}^{\lambda /(t-2)} \rho_{N}^{1-\lambda /(t-2)} \rightarrow 0 \text { as } N \rightarrow \infty .
$$

Later $\left\{\delta_{N}\right\}$ and $\lambda$ will be chosen so that (2.26) holds. If $1 \leqq t^{\prime}<t \leqq 2$, we require

$$
0<\lambda<t-t^{\prime}
$$

Hölder's inequality is applied, as before, and we get

$$
\begin{aligned}
\sum_{k}\left|a_{N, k}\right|^{t-\lambda} & =\sum_{k}\left|a_{N, k}\right|^{t^{\prime}\left(\lambda /\left(t-t^{\prime}\right)\right)}\left|a_{N, k}\right|^{\mid\left(t-t^{\prime}-\lambda\right) /\left(t-t^{\prime}\right)} \\
& \leqq\left(\sum_{k}\left|a_{N, k}\right|^{t^{\prime}}\right)^{\lambda /\left(t-t^{\prime}\right)}\left(\sum_{k}\left|a_{N, k}\right|^{t}\right)^{\left(t-t^{\prime}-\lambda\right) /\left(t-t^{\prime}\right)} \\
& \leqq \alpha_{N}^{\lambda\left(t-t^{\prime}\right)} \rho_{N}^{1-\lambda /\left(t-t^{\prime}\right)} .
\end{aligned}
$$

Hence for Theorems $1 \mathrm{~b}$ and $2 \mathrm{~b}$ it suffices to choose (as we shall do later) $\left\{\delta_{N}\right\}$ and $\lambda$ so that

$$
\delta_{N}^{-(t-\lambda-1)} \alpha_{N}^{\lambda /\left(t-t^{\prime}\right)} \rho_{N}^{1-\lambda /\left(t-t^{\prime}\right)} \rightarrow 0 \text { as } N \rightarrow \infty .
$$

EXPRESSION (2.18). For convenience we let $Z_{N, k}=Y_{N, k}-E Y_{N, k}$. As on page 354 of [6], we use Markov's inequality, with $\nu$ a fixed positive even integer to be chosen later, and obtain

$$
\begin{aligned}
P\left\{\left|\sum_{k} a_{N, k} Z_{N, k}\right|>\varepsilon\right\} & \leqq \frac{1}{\varepsilon^{\nu}} E\left(\sum_{k} a_{N, k} Z_{N, k}\right)^{\nu} \\
& \leqq C \sum_{1} \sum_{2} \prod_{k=1}^{a+b}\left|a_{N, \beta_{k}}\right|^{m_{k} E\left|Z_{N, \beta_{k}}\right|^{m_{k}}}
\end{aligned}
$$

where the first sum is taken over all integers $a, b, m_{1}, \ldots, m_{a+b}$ such that $2 \leqq m_{k}<t$ for $k=1, \ldots, a ; t \leqq m_{k}$ and $2 \leqq m_{k}$ for $k=a+1, \ldots, a+b ; \sum_{k=1}^{a+b} m_{k}=\nu$; and distinct sets of integers $\left\{m_{1}, \ldots, m_{a+b}\right\}$ appear only once in the sum; in the second sum $\beta_{1}, \ldots, \beta_{a+b}$ are allowed to range over the positive integers. Note that the first sum is over a finite number (depending on $\nu$ but not on $N$ ) of terms.

We now proceed to complete the proofs of Theorems 1a, 2a, 3, 4, and 5 by showing that (2.29) is $O\left(\rho_{N}^{1+\xi}\right)$ for some $\xi>0$. For these theorems $E\left|X_{k}\right|^{t} \leqq M<\infty$ for all $k$ which implies that $E\left|Z_{N, k}\right|^{j} \leqq M^{\prime}<\infty$ for all $N, k$ and for $j=1,2, \ldots,[t]$ and $j=t$. Hence if $1 \leqq k \leqq a$

$$
\begin{aligned}
& \left|a_{N, \beta_{k}}\right|^{m_{k} E}\left|Z_{N, \beta_{k}}\right|^{m_{k}} \leqq C\left|a_{N, \beta_{k}}\right|^{2}\left|a_{N, \beta_{k}}\right|^{m_{k}-2} \\
& \leqq C \gamma_{N}^{\left(m_{k}-2\right) / 2} a_{N, \beta_{k}}^{2} \text {, }
\end{aligned}
$$


and if $a+1 \leqq k \leqq a+b$

$$
\begin{aligned}
\left|a_{N, \beta_{k}}\right|^{m_{k}} E\left|Z_{N, \beta_{k}}\right|^{m_{k}} & \leqq\left|a_{N, \beta_{k}}\right|^{t} E\left|Z_{N, \beta_{k}}\right|^{t}\left[\sup _{\omega}\left|a_{N, \beta_{k}} Z_{N, \beta_{k}}(\omega)\right|^{m_{k}-t}\right] \\
& \leqq C \delta_{N^{k}}^{m^{-}-t}\left|a_{N, \beta_{k}}\right|^{t} .
\end{aligned}
$$

Therefore each term in the first sum of (2.29) is bounded by

$$
C \gamma_{N}^{\left(m_{1}+\cdots+m_{a}\right) / 2} \rho_{N}^{b} \delta_{N^{a+1}}^{m_{1}+\cdots+m_{a+b}-b t} .
$$

In Theorems $1 \mathrm{a}$ and $2 \mathrm{a}$ we assume $t<2$ so that $a=0$ in (2.29) and (2.30). Thus (2.30) becomes

$$
C \rho_{N}^{b} \delta_{N}^{v-b t}
$$

For Theorems 1a and 2a we choose $\nu=2$ and $\delta_{N}=\rho_{N}^{1 / 3 t}$ so that $b=1$. This choice of $\delta_{N}$ satisfies (2.23) and (2.24). With this choice of $\delta_{N}$ and $\nu$ expression (2.31) becomes $C \rho_{N}^{1+(\nu-t) / 3 t}$. We note that $\nu-t>0$ and so $1+(\nu-t) / 3 t>1$.

For Theorems 3, 4, and 5 there exists a $\nu_{0}>0$ such that $\gamma_{N}^{\nu_{0}} \leqq O\left(\rho_{N}\right)$. For these theorems we choose $\nu>\max \left(t, 2 v_{0}\right)$ and $\delta_{N}=\rho_{N}^{1 / 3 t}$. This choice of $\delta_{N}$ satisfies (2.23) and (2.24). The proofs of these theorems are completed upon showing that (2.30) is $O\left(\rho_{N}^{1+\xi}\right)$ for some $\xi>0$. If $b=0$ then (2.30) becomes $C \gamma_{N}^{\nu / 2}$ which is of the desired form since $\nu>2 \nu_{0}$. For $b>1$ we note that $\gamma_{N}$ and $\rho_{N} \rightarrow 0$ as $N \rightarrow \infty$, and that $m_{a+1}+\cdots+m_{a+b}-b t \geqq 0$, and thus that (2.30) is of the desired form. If $b=1$ and $a=0$ expression (2.30) becomes $C \rho_{N} \delta_{N}^{v-t}$ which is of the desired form, and if $b=1$ and $a>0$, recalling that $\delta_{N} \rightarrow 0$ as $N \rightarrow \infty, m_{a+1}+\cdots+m_{a+b}-b t \geqq 0$, and $\gamma_{N}^{\nu_{0}}$ $\leqq O\left(\rho_{N}\right)$, we see that $(2.30)$ is $O\left(\rho_{N}^{1+\xi}\right)$ for some $\xi>0$.

The proofs of Theorems $1 \mathrm{~b}, 1 \mathrm{c}, 2 \mathrm{~b}$, and $2 \mathrm{c}$ will be completed by showing (2.29) is $o\left(\rho_{N}\right)$ under the assumption that $y^{t} F(y) \leqq M<\infty$ for all $y>0$. This assumption implies that for a fixed $\lambda$ with $0<\lambda<t-1$,

$$
E\left|Z_{N, k}\right|^{j} \leqq M^{\prime}<\infty \text { for all } N \text { and } k \text { with } j=2, \ldots, t_{0}
$$

and $j=t-\lambda$, where $t_{0}$ is the largest integer with $t_{0}<t$. As in the above, if $1 \leqq k \leqq a$

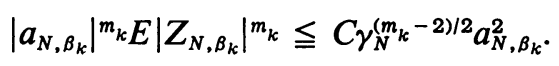

If $a+1 \leqq k \leqq a+b$

$$
\begin{aligned}
\left|a_{N, \beta_{k}}\right|^{m_{k} E} E\left|Z_{N, \beta_{k}}\right|^{m_{k}} & \leqq\left|a_{N, \beta_{k}}\right|^{t-\lambda} E\left|Z_{N, \beta_{k}}\right|^{t-\lambda}\left[\sup _{\omega}\left|a_{N, \beta_{k}} Z_{N, \beta_{k}}\right|^{m_{k}-t+\lambda}\right] \\
& \leqq C \delta_{N}^{m_{k}}-t+\lambda\left|a_{N, \beta_{k}}\right|^{t-\lambda} .
\end{aligned}
$$

So each term in the first sum of (2.29) is bounded by

$$
C \gamma_{N}^{\left(m_{1}+\cdots+m_{a}\right) / 2} \delta_{N^{a+1}}^{m_{1}+\cdots+m_{a+0}-b(t-\lambda)}\left(\sum_{k}\left|a_{N, k}\right|^{t-\lambda}\right)^{b} .
$$

For Theorems $1 \mathrm{c}$ and $2 \mathrm{c}$ where $t>2$ it has been shown earlier that

$$
\sum_{k}\left|a_{N, k}\right|^{t-\lambda} \leqq \gamma_{N}^{\lambda /(t-2)} \rho_{N}^{1-\lambda /(t-2)}
$$


if $0<\lambda<t-2$. Hence (2.32) is bounded by

$$
C \gamma_{N}^{\left(m_{1}+\cdots+m_{a}\right) / 2+b \lambda /(t-2)} \rho_{N}^{b(1-\lambda /(t-2))} \delta_{N}^{m_{a+1}}+\cdots+m_{a+b}-b(t-\lambda) .
$$

Now $\lambda, \nu$, and $\delta_{N}$ are chosen as follows:

$$
\begin{aligned}
0 & <2 \lambda<t-2 \\
\nu & >\max \left(2 t v_{0}, 3 t\right) \\
\delta_{N} & =\max \left(\rho_{N}^{1 / 3 t}, \gamma_{N}^{1 / 2 t}\right) .
\end{aligned}
$$

This choice of $\delta_{N}$ satisfies (2.23). Since $\delta_{N} \rightarrow 0$ as $N \rightarrow \infty$ (2.26) is satisfied if

$$
\delta_{N}^{-t} \gamma_{N}^{\lambda /(t-2)} \rho_{N}^{1-\lambda /(t-2)} \rightarrow 0 \text { as } N \rightarrow \infty \text {. }
$$

But from its definition

$$
\delta_{N}^{-t} \leqq\left(\gamma_{N}^{-(\lambda /(t-2)) / 2 t} \rho_{N}^{-(1-\lambda /(t-2)) / 3 t}\right)^{t}
$$

so (2.34) is obtained. For the two theorems in question it only remains to show (2.33) is $o\left(\rho_{N}\right)$. If $b=0$ expression (2.33) becomes $C \gamma_{N}^{\nu / 2}$ which is $o\left(\rho_{N}\right)$ because $\nu>2 v_{0}$. For $b>1$, expression (2.33) is $o\left(\rho_{N}\right)$ since $\lambda / t-2<1 / 2, \gamma_{N}$ and $\rho_{N} \rightarrow 0$ as $N \rightarrow \infty$, and the exponents on $\gamma_{N}$ and $\rho_{N}$ in (2.33) are nonnegative. If $b=1$, then (2.33) is bounded by

$$
C \delta_{N}^{v}\left(\gamma_{N}^{\left(m_{1}+\cdots+m_{a}\right) / 2} \delta_{N}^{-\left(m_{1}+\cdots+m_{a}\right)}\right)\left(\delta_{N}^{-t} \rho_{N}^{1-\lambda /(t-2)} \gamma_{N}^{\lambda(t-2)}\right) .
$$

Now $\delta_{N}^{v}=o\left(\rho_{N}\right)$,

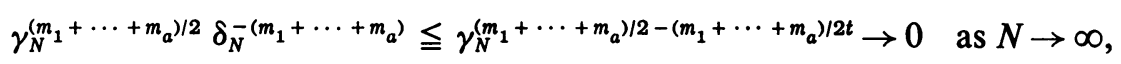

and by (2.34)

$$
\delta_{N}^{-t} \rho_{N}^{1-\lambda /(t-2)} \gamma_{N}^{\lambda /(t-2)} \rightarrow 0 \text { as } N \rightarrow \infty \text {. }
$$

For Theorems $1 \mathrm{~b}$ and $2 \mathrm{~b}$ where $1 \leqq t^{\prime}<t \leqq 2$ it has been shown earlier that

$$
\sum_{k}\left|a_{N, k}\right|^{t-\lambda} \leqq \alpha_{N}^{\lambda\left(t-t^{\prime}\right)} \rho_{N}^{1-\lambda /\left(t-t^{\prime}\right)}
$$

if $0<\lambda<t-t^{\prime}$. We note that for $t \leqq 2$ we have $a=0$ in expression (2.32) which is then bounded by

$$
C \delta_{N}^{\nu-b(t-\lambda)} \alpha_{N}^{b \lambda /\left(t-t^{\prime}\right)} \rho_{N}^{b\left(1-\lambda /\left(t-t^{\prime}\right)\right)}
$$

For these two theorems $\lambda, \nu$, and $\delta_{N}$ are chosen as follows:

$$
\begin{aligned}
0 & <\lambda<2\left(t-t^{\prime}\right) / 3\left(\lambda_{0}+1\right) \\
\nu & >3 t \\
\delta_{N} & =\rho_{N}^{1 / 3 t} .
\end{aligned}
$$

This choice of $\delta_{N}$ satisfies (2.23). Since $\delta_{N} \rightarrow 0$ as $N \rightarrow \infty$ condition (2.28) is satisfied if

$$
\delta_{N}^{-t} \alpha_{N}^{\lambda /\left(t-t^{\prime}\right)} \rho_{N}^{1-\lambda /\left(t-t^{\prime}\right)} \rightarrow 0 \text { as } N \rightarrow \infty
$$


By the choice of $\delta_{N}$ expression (2.37) becomes

$$
\left(\alpha_{N} \rho_{N}^{\left(2 / 3-\lambda /\left(t-t^{\prime}\right)\right)\left(t-t^{\prime}\right) / \lambda}\right)^{\lambda /\left(t-t^{\prime}\right)}
$$

and by the choice of $\lambda$ the exponent on $\rho_{N}$ is greater than $\lambda_{0}$ so (2.37) holds. It only remains to show (2.36) is $o\left(\rho_{N}\right)$. Expression (2.36) can be rewritten as

$$
C \delta_{N}^{v+b \lambda}\left(\delta_{N}^{-t} \alpha_{N}^{\lambda /\left(t-t^{\prime}\right)} \rho_{N}^{1-\lambda /\left(t-t^{\prime}\right)}\right)^{b}
$$

Applying (2.37) and observing that $\delta_{N}^{v}=o\left(\rho_{N}\right)$ the proofs of these theorems are completed.

Now we relax the assumption that $\rho_{N} \rightarrow 0$ as $N \rightarrow \infty$ for Theorems 1a, 1b, and 1c. Define

$$
B_{k}=\left\{b_{N}: P\left\{\left|S_{N}\right|>\varepsilon\right\}=b_{N} \rho_{N} \text { for } N \text { 's such that } 1 /(k+1)<\rho_{N} \leqq 1 / k\right\} .
$$

Now sup $B_{k}$ is finite for each $k$ and if $\sup _{k}\left(\sup B_{k}\right)<\infty$ the proof is complete. If not, that is if

$$
\sup _{k}\left(\sup B_{k}\right)=\infty
$$

then there exists a sequence $\rho_{N_{k}}$ with $\rho_{N_{k}} \rightarrow 0$ as $k \rightarrow \infty$ and $P\left\{\left|S_{N_{k}}\right|>\varepsilon\right\} / \rho_{N_{k}} \rightarrow \infty$ as $k \rightarrow \infty$. This contradiction completes the proof.

3. Further investigation of Theorem 4 and of condition (2.13). We continue using the notation of the previous sections, except that we allow $0<t<1$; in these cases $E\left|X_{N}\right|$ may not be finite for any $N$ so in these cases we define

$$
S_{N}=\sum_{k=1}^{\infty} a_{N, k} X_{k}
$$

and

$$
F(x)=\sup _{k} P\left\{\left|X_{k}\right| \geqq x\right\} .
$$

$F$ will always be a function which could have been obtained via (2.4) or (3.1) depending on whether $t \geqq 1$ or $t<1$ respectively. In the following definitions both $\mathscr{G}$ and $G^{*}$ correspond to a specific $F$, but for convenience we will not use an $F$ subscript in either case.

Let

$$
\mathscr{G}=\left\{\begin{array}{l}
G \mid \begin{array}{l}
G:[0, \infty) \rightarrow[0, \infty) \text { and is nonincreasing } \\
G(x) \geqq F(x) \text { for } 0 \leqq x<\infty \\
G \text { satisfies }(2.11) \text { and }(2.13)
\end{array}
\end{array}\right\}
$$

and define

$$
\begin{aligned}
G^{*}(x) & =\operatorname{Max}\left\{1, \sup _{y \geqq 1} y^{t} F(y)\right\} & & 0 \leqq x \leqq 1 \\
& =\frac{1}{x^{t}} \sup _{y \geqq x} y^{t} F(y) & & 1<x .
\end{aligned}
$$


The results of this section are summarized in the statements of the following propositions and theorems.

Proposition 1. If $\mathscr{G} \neq \varnothing$ then $G^{*} \in \mathscr{G}$. If $G^{*}$ is finite and satisfies (2.11) then $G^{*} \in \mathscr{G}$.

TheOREM 6. If $t>0, \int_{0}^{\infty} y^{t} \log ^{+} y|d F(y)|<\infty$, and $F(y) \rightarrow 0$ as $y \rightarrow \infty$, then $\mathscr{G} \neq \varnothing$. The converse is not true.

Proposition 1 provides a method for checking to see whether the hypotheses of Theorem 4 are satisfied in a particular case. It also is useful in obtaining some of the main results of this section.

Theorem 6 shows that one can ignore the $G$ of Theorem 4 entirely if $\lim _{y \rightarrow \infty} F(y)$ $=0$ and $\int_{0}^{\infty} y^{t} \log ^{+} y|d F(y)|<\infty$. It also shows that Theorem 5 of [4] is a corollary to Theorem 4 of [4]-although showing this to be the case was almost as hard as proving Theorem 5 of [4] directly. The following theorem shows that Theorem 6 is tight in the sense that under any reduction in the moment requirement on $F$, an $F$ can be found such that $\mathscr{G}=\varnothing$.

THEOREM 7. If $t>0$ and $g$ is a nonnegative real valued function on $[0, \infty)$ such that lim $\sup _{x \rightarrow \infty} g(x)=\infty$, then there exists an $F$ such that $\lim _{x \rightarrow \infty} F(x)=0$ and

$$
\int_{0}^{\infty} \frac{x^{t} \log ^{+} x}{g(x)}|d F(x)|<\infty
$$

but such that $\mathscr{G}=\varnothing$.

Theorems 8 and 9 show that slight weakenings of the hypotheses of Theorem 4 allow $\sum_{N=1}^{\infty} N^{\rho-1} P\left\{\left|S_{N}\right|>\varepsilon\right\}=\infty$, and thus show that Theorem 4 and the result obtained by combining it with Theorem 6 are in a sense sharp.

THEOREM 8. If $0<t \leqq 2, \rho>0$, and $F$ (corresponding to $G^{*}$ ) is such that $\lim _{x \rightarrow \infty} F(x)$ $=0$ and such that either $G^{*}(x)=\infty$ for some $x$ or $\int_{0}^{\infty} x^{t}\left|d G^{*}(x)\right|=\infty$, then there exist independent and identically distributed random variables $X_{1}, X_{2}, \ldots$ and coefficients $\left\{a_{N, k}\right\}$ such that $P\left\{\left|X_{k}\right| \geqq x\right\} \leqq F(x)$ for all $x \geqq 0$,

$$
\sum_{k=1}^{\infty}\left|a_{N, k}\right|^{t} \leqq C N^{-\rho},
$$

and

$$
\sum_{N=1}^{\infty} N^{\rho-1} P\left\{\left|S_{N}\right| \geqq 1\right\}=\infty
$$

THEOREM 9. If $t>0, \rho>0$, and $g$ is a nonnegative real-valued function on $[0, \infty)$ such that $\lim \sup _{x \rightarrow \infty} g(x)=\infty$, then there exists an $F$ such that $\lim _{x \rightarrow \infty} F(x)=0$ and

$$
\int_{0}^{\infty} \frac{x^{t} \log ^{+} x}{g(x)}|d F(x)|<\infty
$$


independent and identically distributed random variables $X_{1}, X_{2}, \ldots$ such that $P\left\{\left|X_{k}\right| \geqq x\right\}=F(x)$ for all $x \geqq 0$; and a positive constant $\gamma$ and coefficients $\left\{a_{N, k}\right\}$ such that

and

$$
\sum_{k=1}^{\infty}\left|a_{N, k}\right|^{2} \leqq C N^{-\gamma}, \quad \sum_{k=1}^{\infty}\left|a_{N, k}\right|^{t} \leqq C N^{-\rho}
$$

$$
\sum_{k=1}^{\infty} N^{\rho-1} P\left\{\left|S_{N}\right| \geqq 1\right\}=\infty .
$$

Since for $0<t \leqq 2$ we have

$$
\sum_{k=1}^{\infty}\left|a_{N, k}\right|^{2} \leqq \sum_{k=1}^{\infty}\left|a_{N, k}\right|^{t} \leqq C N^{-\rho}
$$

for large enough $N$, it follows that for $0<t \leqq 2$ Theorem 9 is just a corollary to Theorems 7 and 8.

The rest of this section consists of the proofs of Proposition 1; Theorems 6, 7, and 8; and Theorem 9 when $t>2$.

Proof of Proposition 1. Suppose $G^{*}$ is finite and satisfies (2.11). It is clearly nonincreasing, and using its definition one quickly sees that $G^{*}(x) \geqq F(x)$ for $0 \leqq x<\infty$. Now

$$
\begin{aligned}
\sup _{x \geqq 1} \sup _{y \geqq x} \frac{y^{t} F(y)}{x^{t} G^{*}(x)} & =\max \left\{\frac{\sup _{y \geqq 1} y^{t} F(y)}{\max \left[1, \sup _{y \geqq 1} y^{t} F(y)\right]}, \sup _{x>1} 1\right\} \\
& =1<\infty .
\end{aligned}
$$

Thus $G^{*} \in \mathscr{G}$.

Suppose $G \in \mathscr{G}$ and $\sup _{x \geqq 1} \sup _{y \geqq x} y^{t} F(y) / x^{t} G(x)=\lambda$. If $\lambda \leqq 1$ set $\bar{G}=G$; if $\lambda>1$ set $\bar{G}=\lambda G$. In either case $\bar{G} \in \mathscr{G}$ and

$$
\sup _{x \geq 1} \sup _{y \geqq x} \frac{y^{t} F(y)}{x^{t} \bar{G}(x)} \leqq 1 .
$$

This implies that $G^{*}(x)$ is finite for all $x$, and that $\sup _{y \geqq x} y^{t} F(y) \leqq x^{t} \bar{G}(x)$ for all $x>1$, or equivalently that $G^{*}(x) \leqq \bar{G}(x)$ for all $x>1$. Thus

$$
\lim _{x \rightarrow \infty} G^{*}(x) \leqq \lim _{x \rightarrow \infty} \bar{G}(x)=0
$$

and

$$
\begin{aligned}
\int_{0}^{\infty} y^{t}\left|d G^{*}(y)\right| & \leqq C+\int_{(1, \infty)} y^{t}\left|d G^{*}(y)\right| \\
& \leqq C+\int_{(1, \infty)} y^{t}|d \bar{G}(y)|<\infty .
\end{aligned}
$$

Thus $G^{*}$ is finite and satisfies $(2.11)$ so by the first part of the theorem $G^{*} \in \mathscr{G}$. 
Proof of Theorem 6. Suppose $t>0, F(y) \rightarrow 0$ as $y \rightarrow \infty$, and $\int_{0}^{\infty} y^{t} \log ^{+} y|d F(y)|$ $<\infty$. Define $F^{*}(x)=1$ for $0 \leqq x \leqq 3$, and $F^{*}(x)=F(N+)$ for $N=3,4, \ldots$ and $N<x$ $\leqq N+1$. Then $F^{*}(x) \rightarrow 0$ as $x \rightarrow \infty$ and $F^{*}$ can be thought of as the "tail" of a distribution function. In addition $F^{*}(x) \geqq F(x)$ for all $x$ and

$$
\begin{aligned}
\int_{0}^{\infty} y^{t} \log ^{+} y\left|d F^{*}(y)\right| \\
\quad=3^{t} \log 3[1-F(3+)]+\sum_{k=4}^{\infty} k^{t} \log k[F(k-1+)-F(k+)] \\
\quad \leqq C+\sum_{k=3}^{\infty}\left[\left(\frac{k+1}{k}\right)^{t} \frac{\log (k+1)}{\log k}\right] k^{t} \log k[F(k+)-F(k+1+)] \\
\quad \leqq C+\left[\left(\frac{4}{3}\right)^{t} \frac{\log 4}{\log 3}\right] \sum_{k=3}^{\infty} k^{t} \log k[F(k+)-F(k+1+)] \\
\leqq C+C \int_{0}^{\infty} y^{t} \log ^{+} y|d F(y)|<\infty .
\end{aligned}
$$

Let $G^{*}$ correspond to $F$ and $H^{*}$ correspond to $F^{*}$, using (3.3) to define $G^{*}$ and $H^{*}$. Since $F^{*}(x) \geqq F(x)$ for all $x$ we see that $H^{*}(x) \geqq G^{*}(x)$ for all $x$. Now

$$
\int_{0}^{\infty} y^{t} \log ^{+} y\left|d F^{*}(y)\right|<\infty
$$

implies $y^{t} F^{*}(y) \rightarrow 0$ as $y \rightarrow \infty$. Thus $H^{*}$ (and also $G^{*}$ ) is finite and converges to zero as $y \rightarrow \infty$. We will show that $\int_{0}^{\infty} y^{t}\left|d H^{*}(y)\right|<\infty$ which, since

$$
\int_{0}^{\infty} y^{t}\left|d G^{*}(y)\right| \leqq \int_{0}^{\infty} y^{t}\left|d H^{*}(y)\right|,
$$

will be enough to guarantee that $G^{*}$ satisfies (2.11) and, from Proposition 1 that $G^{*} \in \mathscr{G}$.

If $F^{*}(x)=0$ for $x \geqq N$ then $H^{*}(x)=0$ for $x \geqq N$ so $\int_{0}^{\infty} y^{t}\left|d H^{*}(y)\right|<\infty$ and we are done.

Assume otherwise, i.e. that $F^{*}(x)>0$ for $0 \leqq x<\infty$. Note that $F^{*}$ is left continuous with all its points of decrease at discontinuities and with an infinite number of discontinuities all contained in the set $\{3,4, \ldots\}$; that $y^{t} F^{*}(y)$ is left continuous, is strictly increasing in every interval $(N, N+1]$ for $N \geqq 3$, and has local maxima contained in the set $\{3,4, \ldots\}$; and thus that there exist integers $3=x_{0}<x_{1}$ $<x_{2}<\cdots$ such that

(a) $\sup _{y \geqq x} y^{t} F^{*}(y)=x_{k}^{t} F^{*}\left(x_{k}\right)$ for $x_{k-1}<x$ and $k=1,2, \ldots$;

(b) $x_{1}^{t} F^{*}\left(x_{1}\right)>x_{2}^{t} F^{*}\left(x_{2}\right)>\cdots$;

$$
\begin{aligned}
H^{*}(x) & =\max \left\{1, \sup _{y \geqq 1} y^{t} F^{*}(y)\right\} & & 0 \leqq x \leqq 1 \\
\text { (c) } \quad & & =\frac{1}{x^{t}} \sup _{y \geqq 1} y^{t} F^{*}(y) & 1 \leqq x \leqq 3 \\
& =\frac{1}{x^{t}} x_{k}^{t} F^{*}\left(x_{k}\right) & & x_{k-1}<x \leqq x_{k} \text { and } k=1,2, \ldots ;
\end{aligned}
$$


(d) $H^{*}\left(x_{k}\right)=F^{*}\left(x_{k}\right)$ for $k=1,2, \ldots$

Define $p_{k}=F^{*}\left(x_{k}\right)-F^{*}\left(x_{k+1}\right)$ and note that $\sum_{k=1}^{\infty} x_{k}^{t} \log x_{k} p_{k}<\infty$. Now

$$
\begin{aligned}
\int_{0}^{\infty} y^{t}\left|d H^{*}(y)\right|= & \int_{\left(0, x_{1}\right)} y^{t}\left|d H^{*}(y)\right|+\sum_{k=1}^{\infty} x_{k \mid}^{t} \Delta H^{*}\left(x_{k}\right) \mid \\
& +\sum_{k=2}^{\infty} \int_{\left(x_{k-1}, x_{k}\right)} y^{t}\left|d H^{*}(y)\right|
\end{aligned}
$$

The first expression on the right-hand side of (3.4) is finite. The second expression is easily seen to be finite as follows:

$$
\begin{aligned}
\sum_{k=1}^{\infty} x_{k}^{t}\left|\Delta H^{*}\left(x_{k}\right)\right| & =\sum_{k=1}^{\infty} x_{k}^{t}\left[F^{*}\left(x_{k}\right)-\left(\frac{x_{k+1}}{x_{k}}\right)^{t} F^{*}\left(x_{k+1}\right)\right] \\
& \leqq \sum_{k=1}^{\infty} x_{k}^{t} p_{k}<\infty .
\end{aligned}
$$

The third expression on the right-hand side of (3.4) is a little more difficult to deal with.

Define $\mathscr{K}=\left\{k \mid k\right.$ is a positive integer and $\left.F^{*}\left(x_{k}\right) / p_{k} \leqq 2\right\}=\left\{k_{1}<k_{2}<\cdots\right\}$. Then

$$
\begin{aligned}
\sum_{k \geqq 1 ; k \notin \mathscr{X}} \int_{\left(x_{k}, x_{k+1}\right)} y^{t}\left|d H^{*}(y)\right| & \leqq \sum_{k \geqq 1 ; k \notin \mathscr{X}} x_{k+1}^{t} \int_{\left(x_{k}, x_{k+1}\right)}\left|d H^{*}(y)\right| \\
& \leqq \sum_{k \geqq 1 ; k \notin \mathscr{X}} x_{k+1}^{t} \int_{\left[x_{k}, x_{k+1}\right)}\left|d F^{*}(y)\right| \\
& \leqq \sum_{k \geqq 1 ; k \notin \mathscr{X}}\left(\frac{x_{k+1}}{x_{k}}\right)^{t} \int_{\left[x_{k}, x_{k+1}\right)} x^{t}\left|d F^{*}(x)\right| .
\end{aligned}
$$

Since $x_{k+1}^{t} F^{*}\left(x_{k+1}\right)<x_{k}^{t} F^{*}\left(x_{k}\right)$ for $k \geqq 1$ we see that

$$
\left(\frac{x_{k+1}}{x_{k}}\right)^{t}<\frac{F^{*}\left(x_{k}\right)}{F^{*}\left(x_{k}\right)-p_{k}}=\frac{1}{1-\left[p_{k} / F^{*}\left(x_{k}\right)\right]}<2
$$

when $F^{*}\left(x_{k}\right) / p_{k}>2$ so that (3.5) is bounded by $2 \int_{0}^{\infty} x^{t}\left|d F^{*}(x)\right|$ which is finite.

Also

$$
\begin{aligned}
\sum_{k \in \mathscr{K}} \int_{\left(x_{k}, x_{k+1}\right)} y^{t}\left|d H^{*}(y)\right| & =\sum_{k \in \mathscr{X}} t x_{k+1}^{t} F^{*}\left(x_{k+1}\right) \int_{x_{k}}^{x_{k+1}} \frac{1}{y} d y \\
& \leqq \sum_{k \in \mathscr{X}} t x_{k+1}^{t} F^{*}\left(x_{k+1}\right) \log x_{k+1} \\
& =t \sum_{i} x_{k_{i}+1}^{t} \log x_{k_{i}+1}\left[p_{k_{i}+1}+\cdots+p_{k_{i+1}-1}+F^{*}\left(x_{k_{i+1}}\right)\right] \\
& \leqq t \sum_{i}\left[\sum_{k=k_{i}+1}^{k_{i+1}-1} x_{k}^{t} \log x_{k} p_{k}+2 x_{k_{i+1}}^{t} \log x_{k_{i+1}} p_{k_{i+1}}\right] \\
& \leqq 2 t \sum_{k=1}^{\infty} x_{k}^{t} \log x_{k} p_{k}<\infty
\end{aligned}
$$


Since both (3.5) and (3.6) are finite, we have finished showing that (3.4) is finite and thus completed the proof of the main part of the theorem.

The following example shows that the converse is false. Let

$$
\begin{aligned}
F(x) & =1 & & 0 \leqq x \leqq 2 \\
& =C \int_{x}^{\infty} y^{-t-1}(\log y)^{-2} d y & & x \geqq 2
\end{aligned}
$$

where $t>0$ and $C=\left[\int_{2}^{\infty} x^{-t-1}(\log x)^{-2} d x\right]^{-1}$. In this case $x^{t} F(x)$ is strictly decreasing for $x \geqq 2$ from which it follows that $G^{*}=F_{2}$. Then

$$
\int_{0}^{\infty} y^{t} \log ^{+} y|d F(y)|=\int_{2}^{\infty} \frac{1}{y \log y} d y=\infty
$$

but $\lim _{y \rightarrow \infty} G^{*}(y)=0$ and

$$
\int_{0}^{\infty} y^{t}\left|d G^{*}(y)\right|=\int_{1}^{\infty} \frac{1}{y(\log y)^{2}} d y+C<\infty .
$$

In this case $G^{*} \in \mathscr{G}$ but $\int_{0}^{\infty} y^{t} \log ^{+} y|d F(y)|$ is not finite.

Proof of Theorem 7. Fix $\alpha>0, t>0$ and choose $3<x_{1}<x_{2}<\cdots$ such that

(1) $g\left(x_{k}\right) \geqq k^{\alpha}$ for $k=1,2, \ldots$;

(2) $\log x_{k+1} \geqq 2 \log x_{k}$ for $k=1,2, \ldots$.

Define

$$
p_{k}=\mu g\left(x_{k}\right) / k^{1+\alpha} \max \left\{x_{k}^{t} \log x_{k}, g\left(x_{k}\right)\right\}
$$

where $\mu$ is such that $\sum_{k=1}^{\infty} p_{k}=1$, and define $F(x)=\sum_{\left\{k: x_{k} \geqq x\right\}} p_{k} . F$ can be thought of as the "tail" of the distribution of a random variable $X$ such that $P\left\{X= \pm x_{k}\right\}$ $=p_{k} / 2$. Obviously $F(x) \rightarrow 0$ as $x \rightarrow \infty$.

$$
\begin{aligned}
\int_{0}^{\infty} \frac{x^{t} \log ^{+} x}{g(x)}|d F(x)| & =\sum_{k=1}^{\infty} \frac{x_{k}^{t} \log x_{k}}{g\left(x_{k}\right)} p_{k} \\
& =\mu \sum_{k=1}^{\infty} \frac{x_{k}^{t} \log x_{k}}{k^{1+\alpha} \max \left\{x_{k}^{t} \log x_{k}, g\left(x_{k}\right)\right\}} \\
& \leqq \mu \sum_{k=1}^{\infty} \frac{1}{k^{1+\alpha}}<\infty
\end{aligned}
$$

However

$$
\begin{aligned}
\int_{0}^{\infty} x^{t}\left|d G^{*}(x)\right| & \geqq \sum_{k=1}^{\infty} \int_{\left(x_{k}, x_{k+1}\right)} x^{t}\left|d G^{*}(x)\right| \\
& =\sum_{k=1}^{\infty} \int_{\left(x_{k}, x_{k+1}\right)} \frac{t}{x}\left[\sup _{y \geqq x_{k+1}} y^{t} F(y)\right] d x \\
& \geqq \sum_{k=1}^{\infty} t x_{k+1}^{t} p_{k+1} \log \frac{x_{k+1}}{x_{k}}
\end{aligned}
$$




$$
\begin{aligned}
& \geqq \frac{t}{2} \sum_{k=1}^{\infty} x_{k+1}^{t} \log x_{k+1} p_{k+1} \\
& =C \sum_{k=2}^{\infty} \frac{x_{k}^{t} g\left(x_{k}\right) \log x_{k}}{k^{1+\alpha} \max \left\{x_{k}^{t} \log x_{k}, g\left(x_{k}\right)\right\}} \\
& =C \sum_{k=1}^{\infty} \frac{\min \left\{x_{k}^{t} \log x_{k}, g\left(x_{k}\right)\right\}}{k^{1+\alpha}} \\
& =\infty .
\end{aligned}
$$

Thus $G^{*}$ does not satisfy (2.11) and from Proposition 1 it follows that $\mathscr{G}$ is empty.

Lemma. If $A_{1}, \ldots, A_{M}$ are measurable subsets of a probability space, then

$$
P\left(\bigcup_{k=1}^{M} A_{k}\right) \geqq \sum_{k=1}^{M} P\left(A_{k}\right)-\sum_{1 \leqq j<k \leqq M} P\left(A_{j} \cap A_{k}\right) .
$$

Proof. Is simple using induction.

\section{Proof of Theorem 8. Define}

$$
\begin{aligned}
F_{0}(x) & =1 & & x=0 \\
& =F(2-) & & 0<x \leqq 1 \\
& =F\left(2^{N+1}-\right) & & 2^{N-1}<x \leqq 2^{N} \text { and } N=1,2, \ldots,
\end{aligned}
$$

and note that $F_{0}$ is left continuous. Let $X_{1}, X_{2}, \ldots$ be a sequence of independent and identically distributed random variables which are symmetrically distributed about zero and satisfy $P\left\{\left|X_{k}\right| \geqq x\right\}=F_{0}(x)$ for $x \geqq 0$. Define

$$
\begin{aligned}
a_{N, k} & =a_{N} & & 1 \leqq k \leqq \nu_{N} \\
& =0 & & k>\nu_{N}
\end{aligned}
$$

where $\left\{a_{N}\right\}$ and $\left\{\nu_{N}\right\}$ will be obtained later. Define

$$
S_{N, k}=S_{N}-a_{N} X_{k} \quad \text { for } 1 \leqq k \leqq \nu_{N}
$$

and

$$
A_{N, k}=\left\{S_{N, k} \geqq 0, a_{N} X_{k} \geqq 1\right\} \quad 1 \leqq k \leqq \nu_{N} .
$$

Then from the preceding lemma

$$
\begin{aligned}
P\left\{\left|S_{N}\right| \geqq 1\right\} & \geqq P\left(\bigcup_{k=1}^{v_{N}} A_{N, k}\right) \\
& \geqq \sum_{k=1}^{\nu_{N}} P\left(A_{N, k}\right)-\sum_{1 \leqq j<k \leqq v_{N}} P\left(A_{N, j} \cap A_{N, k}\right) \\
& \geqq \frac{\nu_{N}}{4} F_{0}\left(\frac{1}{a_{N}}\right)-\frac{\nu_{N}^{2}}{8}\left[F_{0}\left(\frac{1}{a_{N}}\right)\right]^{2} \\
& \geqq \frac{\nu_{N}}{4} F_{0}\left(\frac{1}{a_{N}}\right)\left[1-\nu_{N} F_{0}\left(\frac{1}{a_{N}}\right)\right] .
\end{aligned}
$$


If $\lim \sup _{x \rightarrow \infty} x^{t} F(x) \neq 0$, choose $\left\{a_{N}\right\}$ such that $0<a_{N} \leqq N^{-2 \rho / t}$ and such that

$$
\inf _{N}\left(\frac{1}{a_{N}}\right)^{t} F_{0}\left(\frac{1}{a_{N}}\right)>0
$$

and choose

$$
\nu_{N}=\min \left\{\left[N^{-\rho} a_{N}^{-t}\right],\left[1 / 2 F_{0}\left(\frac{1}{a_{N}}\right)\right]\right\}
$$

where $[y]$ is the integral part of $y$. Then

$$
\frac{\nu_{N}}{4} F_{0}\left(\frac{1}{a_{N}}\right)\left[1-\nu_{N} F_{0}\left(\frac{1}{a_{N}}\right)\right] \geqq \frac{1}{8} \nu_{N} F_{0}\left(\frac{1}{a_{N}}\right) .
$$

Since $F_{0}\left(1 / a_{N}\right) \rightarrow 0$ as $N \rightarrow \infty$, it follows that $\left[1 / 2 F_{0}\left(1 / a_{N}\right)\right] \rightarrow \infty$ and

$$
\left[1 / 2 F_{0}\left(1 / a_{N}\right)\right] F_{0}\left(1 / a_{N}\right) \rightarrow \frac{1}{2} \quad \text { as } N \rightarrow \infty
$$

Also $\left[N^{-\rho} a_{N}^{-t}\right] \geqq\left[N^{\rho}\right]$ and

$$
\left[N^{-\rho} a_{N}^{-t}\right] F_{0}\left(\frac{1}{a_{N}}\right)=\left\{a_{N}^{t}\left[N^{-\rho} a_{N}^{-t}\right]\right\}\left(\frac{1}{a_{N}}\right)^{t} F_{0}\left(\frac{1}{a_{N}}\right) \geqq C N^{-\rho} .
$$

Thus from (3.8)-(3.10) and the preceding discussion of the asymptotic behavior of $F_{0}\left(1 / a_{N}\right)$ times each of the possible expressions for $\nu_{N}$, it follows that for large enough $N, P\left\{\left|S_{N}\right| \geqq 1\right\} \geqq C N^{-\rho}$ and

$$
\sum_{N=1}^{\infty} N^{\rho-1} P\left\{\left|S_{N}\right| \geqq 1\right\} \geqq \sum_{N=C}^{\infty} C N^{-1}=\infty
$$

Now suppose $\lim \sup _{x \rightarrow \infty} x^{t} F(x)=0$. Then if we choose $\left\{\nu_{N}\right\}$ and $\left\{a_{N}\right\}$ so that $\nu_{N} a_{N}^{t} \leqq N^{-\rho}$ we will have

$$
\nu_{N} F_{0}\left(\frac{1}{a_{N}}\right)=\left(\nu_{N} a_{N}^{t}\right)\left\{\left(\frac{1}{a_{N}}\right)^{t} F_{0}\left(\frac{1}{a_{N}}\right)\right\}
$$

which converges to zero as $N \rightarrow \infty$ so that from (3.8), for sufficiently large $N$

$$
P\left\{\left|S_{N}\right| \geqq 1\right\} \geqq \frac{1}{8} v_{N} F_{0}\left(1 / a_{N}\right)
$$

Since $x^{t} F(x) \rightarrow 0$ as $N \rightarrow \infty$ we see that $G^{*}(x)$ is finite for all $x$. Since $F_{0}(x) \leqq F(x)$ we see that if $G_{0}$ corresponds to $F_{0}$, then $G_{0}(x)$ is also finite for all $x$. In addition, for $x>4$ we have $F(x) \leqq F_{0}(x / 4)$ so that $G^{*}(x) \leqq G_{0}(x / 4)$. Thus $\int_{0}^{\infty} x^{t}\left|d G^{*}(x)\right|=\infty$ implies $\int_{0}^{\infty} x^{t}\left|d G_{0}(x)\right|=\infty$. Let $x_{1}<x_{2}<\cdots$ be those points (denumerably many of them) for which $x_{1}>4$ and $F_{0}\left(x_{i}\right)=G_{0}\left(x_{i}\right)$ for all $i$. Note that $x_{i}=2^{k}$ for some positive 
integer $k$ and thus that $x_{i+1} \geqq 2 x_{i}$ for all $i$. Define $p_{k}=F_{0}\left(x_{k}\right)-F_{0}\left(x_{k+1}\right)$ and note that for $x_{k}<x \leqq x_{k+1}$ we have $G_{0}(x)=\left(1 / x^{t}\right) x_{k+1}^{t} F_{0}\left(x_{k+1}\right)$. Then

$$
\begin{aligned}
\int_{0}^{\infty} x^{t}\left|d G_{0}(x)\right|= & C+\sum_{k=1}^{\infty} \int_{\left(x_{k}, x_{k}+1\right)} x^{t}\left|d G_{0}(x)\right| \\
& +\sum_{k=1}^{\infty} x_{k}^{t}\left[F_{0}\left(x_{k}\right)-\left(\frac{x_{k+1}}{x_{k}}\right)^{t} F_{0}\left(x_{k+1}\right)\right] \\
\leqq & C+\sum_{k=1}^{\infty} \int_{x_{k}}^{x_{k+1}} \frac{t}{x} x_{k+1}^{t} F_{0}\left(x_{k+1}\right) d x+\sum_{k=1}^{\infty} x_{k}^{t} p_{k} \\
= & C+t \sum_{k=2}^{\infty} x_{k}^{t} F_{0}\left(x_{k}\right) \log \left(\frac{x_{k}}{x_{k-1}}\right)+\sum_{k=1}^{\infty} x_{k}^{t} p_{k} .
\end{aligned}
$$

Recall that $x_{k} \geqq 2 x_{k-1}$ so that

$$
\sum_{k=2}^{\infty} x_{k}^{t} F_{0}\left(x_{k}\right) \log \left(\frac{x_{k}}{x_{k-1}}\right) \geqq C \sum_{k=2}^{\infty} x_{k}^{t} F_{0}\left(x_{k}\right) \geqq C \sum_{k=2}^{\infty} x_{k}^{t} p_{k} .
$$

Thus $\int_{0}^{\infty} x^{t}\left|d G_{0}(x)\right|=\infty$ implies

$$
\sum_{k=2}^{\infty} x_{k}^{t} F_{0}\left(x_{k}\right) \log \left(\frac{x_{k}}{x_{k-1}}\right)=\infty
$$

Now let

$$
\begin{array}{ll}
N_{k}=\left[x_{k}^{t / \rho}\right] & \text { for } k \geqq 1 \text { and } N_{0}=0, \\
a_{N}=1 / x_{k} & \text { for } N_{k-1}<N \leqq N_{k},
\end{array}
$$

and

$$
\nu_{N}=\left[N^{-\rho} a_{N}^{-t}\right] \text { for all } N .
$$

Note that $\nu_{N_{k}}=1$ for all $k$ and $\nu_{N} \geqq 1$ for all other $N$, also that $\nu_{N} a_{N}^{t} \leqq N^{-\rho}$. We thus have from (3.11)

$$
\begin{aligned}
\sum_{N=1}^{\infty} N^{\rho-1} P\left\{\left|S_{N}\right| \geqq 1\right\} & \geqq C \sum_{N=1}^{\infty} N^{\rho-1} \nu_{N} F_{0}\left(\frac{1}{a_{N}}\right) \\
& =C \sum_{k=1}^{\infty} F_{0}\left(x_{k}\right) \sum_{N_{k-1}<N \leqq N_{k}} N^{\rho-1} \nu_{N} \\
& \geqq C \sum_{k=1}^{\infty} F_{0}\left(x_{k}\right) x_{k}^{t} \sum_{N=N_{k-1+1}}^{N_{k}} N^{-1} \\
& \geqq C \sum_{k=1}^{\infty} x_{k}^{t} F_{0}\left(x_{k}\right) \log \frac{N_{k}}{N_{k-1}} \\
& \geqq C \sum_{k=C}^{\infty} x_{k}^{t} F_{0}\left(x_{k}\right) \log \left(\frac{x_{k}}{x_{k-1}}\right)=\infty
\end{aligned}
$$


Proof of Theorem 9 for $t>2$. Let $\left\{y_{k}\right\}$ be an increasing sequence of positive real numbers such that $g\left(y_{k}\right) \geqq 2^{k}$ for all $k$, such that $y_{k} \geqq 2^{k}$, and such that either

(1) $g\left(y_{k}\right) \geqq y_{k}^{t} \log y_{k}$ for all $k$, or

(2) $g\left(y_{k}\right)<y_{k}^{t} \log y_{k}$ for all $k$.

Case 1. Suppose $g\left(y_{k}\right) \geqq y_{k}^{t} \log y_{k}$ for all $k$. Fix $\alpha>0$ and define

$$
p_{k}=\mu / y_{k} k^{1+\alpha} \text { for } k=1,2, \ldots
$$

where $\mu$ is such that $\sum_{k=1}^{\infty} p_{k}=1$, let

$$
F(x)=\sum_{\left\{k: y_{k} \geqq x\right\}} p_{k} \text { for } x \geqq 0,
$$

and suppose $X_{1}, X_{2}, \ldots$ are independent and identically distributed random variables such that $P\left\{X_{n}= \pm y_{k}\right\}=p_{k} / 2$ for $k=1,2, \ldots$. Note that $E\left|X_{n}\right|<\infty$, $E X_{n}=0$, and

$$
\int_{0}^{\infty} \frac{x^{t} \log ^{+} x}{g(x)}|d F(x)|=\sum_{k=1}^{\infty} \frac{y_{k}^{t} \log y_{k}}{g\left(y_{k}\right)} p_{k} \leqq \sum_{k=1}^{\infty} p_{k}=1 .
$$

Using some of the notation of the first part of the proof of Theorem 8 , let $\nu_{n} \equiv 1$ and $a_{N, 1}=N^{-\rho / t}$. Then

$$
\sum_{k=1}^{\infty}\left|a_{N, k}\right|^{t}=N^{-\rho}, \quad \sum_{k=1}^{\infty}\left|a_{N, k}\right|^{2}=N^{-2 \rho / t}
$$

and

$$
\begin{aligned}
\sum_{N=1}^{\infty} N^{\rho-1} P\left\{\left|S_{N}\right| \geqq 1\right\} & =\sum_{N=1}^{\infty} N^{\rho-1} P\left\{\left|X_{1}\right| \geqq N^{\rho / t}\right\} \\
& \geqq C E\left|X_{1}\right|^{t}=C \sum_{k=1}^{\infty} \frac{y_{k}^{t-1}}{k^{1+\alpha}} \\
& \geqq C \sum_{k=1}^{\infty} \frac{2^{k(t-1)}}{k^{1+\alpha}}=\infty
\end{aligned}
$$

Case 2. Suppose $g\left(y_{k}\right)<y_{k}^{t} \log y_{k}$ for all $k$. Let $0<\varepsilon<1 / t$ and define $\lambda_{1}=$ $(t-2 t \varepsilon) /(t-2)$ and $\lambda_{2}=(t-t \varepsilon) /(t-2)$. Note that $1<\lambda_{1}<\lambda_{2}$. We want a sequence $\left\{x_{n}\right\}$ of positive real numbers such that

(1) $\left\{x_{n}\right\}$ contains an infinite number of members of $\left\{y_{k}\right\}$,

(2) $\lambda_{1} \leqq \log x_{n+1} / \log x_{n} \leqq \lambda_{2}$ for $n=1,2, \ldots$.

Let $x_{0}=y_{i_{0}}$ for some $y_{i_{0}}>1$. Choose $\nu \geqq 1$ such that $\lambda_{1}^{\nu}<\lambda_{2}^{\nu-1}$ and choose $y_{i_{1}}$ $\geqq x_{0}^{\lambda \nu}$. There exists $m_{1} \geqq \nu$ such that $x_{0^{1}}^{\lambda_{1}}<x_{0^{2}}^{m_{1}-1} \leqq y_{i_{1}}<x_{0^{2}}^{m_{1}}$. Because $x_{0}^{\lambda_{1} m_{1}}$ is continuous in $\lambda$, a value of $\lambda$, say $\lambda_{1}^{*}$, exists such that $\lambda_{1}<\lambda_{1}^{*}<\lambda_{2}$ and $y_{i_{1}}=x_{0}^{\left.\left(\lambda_{1}^{*}\right)^{m}\right)_{1}}$. Define $n_{0}=0, n_{1}=m_{1}$, and $x_{k}=x_{0}^{\left(\lambda_{1}^{*}\right)^{k}}$ for $1 \leqq k \leqq m_{1}$. Now starting with $x_{n_{1}}$ instead of $x_{0}$ we repeat this procedure to obtain $y_{i_{2}} \geqq x_{n_{1}^{2}}^{\lambda{ }^{2}-1}$, an integer $m_{2} \geqq \nu$ such that

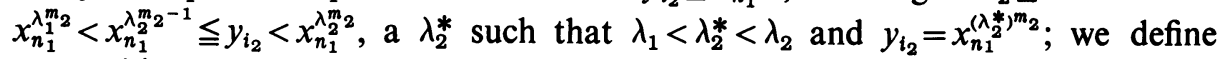
$x_{k}=x_{n_{1}}^{\left(\lambda_{2}^{*}\right)^{k-n_{1}}}$ for $n_{1}+1 \leqq k \leqq n_{1}+m_{2}=n_{2}$. Performing the obvious induction we 
obtain the desired sequence $\left\{x_{k}\right\}$ and an increasing sequence $\left\{n_{k}\right\}$ such that $x_{n_{k}}$ is a member of $\left\{y_{i}\right\}$ for each $k$. Let $\Gamma=\left\{n_{1}, n_{2}, \ldots\right\}$, let $\beta>0$, and define

$$
\begin{aligned}
p_{n} & =\frac{\alpha \min \left\{g\left(x_{n}\right), \log x_{n}\right\}}{n^{1+\beta} x_{n}^{t} \log x_{n}} & & n \notin \Gamma, \\
& =\frac{\alpha}{x_{n}^{t} \log x_{n}} & & n \in \Gamma,
\end{aligned}
$$

where $\alpha$ is such that $\sum_{n=1}^{\infty} p_{n}=1$. Define $F(x)=\sum_{\left(k: x_{k} \geqq x\right)} p_{k}$ for $x \geqq 0$ and suppose $X_{1}, X_{2}, \ldots$ are independent and identically distributed random variables such that $P\left\{X_{n}= \pm x_{k}\right\}=p_{k} / 2$ for $k=1,2, \ldots$ Note that

$$
\begin{aligned}
E\left|X_{k}\right|^{t} & =\alpha \sum_{n \in \Gamma} \frac{\min \left\{g\left(x_{n}\right), \log x_{n}\right\}}{n^{1+\beta} \log x_{n}}+\alpha \sum_{n \in \Gamma} \frac{1}{\log x_{n}} \\
& \leqq \alpha \sum_{n=1}^{\infty} \frac{1}{n^{1+\beta}}+\alpha \sum_{n=1}^{\infty} \frac{1}{\left(\log x_{1}\right) \lambda_{1}^{n-1}}<\infty
\end{aligned}
$$

and that

$$
\begin{aligned}
E \frac{\left|X_{k}\right|^{t} \log ^{+}\left|X_{k}\right|}{g\left(\left|X_{k}\right|\right)} & =\alpha \sum_{n \in \Gamma} \frac{\min \left\{g\left(x_{n}\right), \log x_{n}\right\}}{n^{1+\beta} g\left(x_{n}\right)}+\alpha \sum_{n \in \Gamma} \frac{1}{g\left(x_{n}\right)} \\
& \leqq \alpha \sum_{n=1}^{\infty} \frac{1}{n^{1+\beta}}+\alpha \sum_{k=1}^{\infty} \frac{1}{g\left(x_{n_{k}}\right)} \\
& \leqq C+\alpha \sum_{k=1}^{\infty} \frac{1}{2^{k}}<\infty .
\end{aligned}
$$

Using the notation of the first part of the proof of Theorem 8 we set

$$
\begin{aligned}
& N_{0}=0 \text { and } N_{k}=\left[x_{k}^{t / \rho}\right] \text { for } k=1,2, \ldots \\
& a_{N}=1 / x_{k} \text { for } N_{k-1}<N \leqq N_{k} \text { and } k=1,2, \ldots \\
& \nu_{N}=\left[N^{-\rho} a_{N}^{-t}\right]
\end{aligned}
$$

and note that $\sum_{k=1}^{\infty}\left|a_{N, k}\right|^{t}=\nu_{N} a_{N}^{t} \leqq N^{-\rho}$,

$$
\begin{aligned}
\sum_{k=1}^{\infty}\left|a_{N, k}\right|^{2} & =\nu_{N} a_{N}^{2} \leqq N^{-\rho \varepsilon} N_{k-1}^{-\rho(1-\varepsilon)} x_{k}^{t-2} \\
& \leqq C N^{-\rho \varepsilon} x_{k-1}^{-t(1-\varepsilon)} x_{k}^{t-2} \\
& =C N^{-\rho \varepsilon} \exp \left\{(t-2) \log x_{k-1}\left[-\frac{t(1-\varepsilon)}{t-2}+\frac{\log x_{k}}{\log x_{k-1}}\right]\right\} \\
& \leqq C N^{-\rho \varepsilon}
\end{aligned}
$$

since by construction of the $x_{k}$ 's the exponent of $e$ above is negative; we define $\gamma=\rho \varepsilon$. Now $E\left|X_{k}\right|^{t}<\infty$ implies $x^{t} F(x) \rightarrow 0$ as $x \rightarrow \infty$ so referring to the arguments of (3.7), (3.8), and (3.10) we have for large enough $N$

$$
P\left\{\left|S_{N}\right| \geqq 1\right\} \geqq \frac{1}{8} \nu_{N} F\left(1 / a_{N}\right)
$$


so that

$$
\begin{aligned}
\sum_{N=1}^{\infty} N^{\rho-1} P\left\{\left|S_{N}\right| \geqq 1\right\} & \geqq C \sum_{N=C}^{\infty} N^{\rho-1} \nu_{N} F\left(\frac{1}{a_{N}}\right) \\
& \geqq C \sum_{k=C}^{\infty} x_{k}^{t} F\left(x_{k}\right) \sum_{N=N_{k-1}+1}^{N_{k}} N^{-1} \\
& \geqq C \sum_{k=C}^{\infty} x_{k}^{t} F\left(x_{k}\right) \log \frac{N_{k}}{N_{k-1}} \\
& \geqq C \sum_{k=C}^{\infty} x_{k}^{t} p_{k} \log \frac{x_{k}}{x_{k-1}}
\end{aligned}
$$

Now $x_{k-1} \leqq x_{k}^{1 / \lambda_{1}}$ so that $x_{k} / x_{k-1} \geqq x_{k}^{1-1 / \lambda_{1}}$ and thus

$$
\begin{aligned}
\sum_{N=1}^{\infty} N^{\rho-1} P\left\{\left|S_{N}\right| \geqq 1\right\} & \geqq C \sum_{k=C}^{\infty} x_{k}^{t} p_{k} \log x_{k} \\
& \geqq C \sum_{k=C}^{\infty} x_{n_{k}}^{t} p_{n_{k}} \log x_{n_{k}} \\
& =C \sum_{k=C}^{\infty} \alpha=\infty .
\end{aligned}
$$

4. Concluding remarks. Results for $t<1$ and results of the sharpness of Theorems 1, 2, 3, and 5 are of some interest though investigations of these matters should be fairly routine.

For $t \leqq 2$ we see that

$$
\sum_{k=1}^{\infty}\left|a_{N, k}\right|^{2} \leqq\left[\max _{k}\left|a_{N, k}\right|\right]^{2-t} \sum_{k=1}^{\infty}\left|a_{N, k}\right|^{t}
$$

so that $\gamma_{N} \rightarrow 0$ at least as fast as $\rho_{N}$ does. So for $t \leqq 2$ no assumption need be made on the rate at which $\gamma_{N} \rightarrow 0$. (In Theorems 4 and 5 the assumption $\gamma>0$ is automatically satisfied if $t \leqq 2$.) Suppose $t>2$, the $X_{k}$ 's are independent random variables normally distributed with common mean zero and common variance one, and $\sum_{k=1}^{\infty}\left|a_{N, k}\right|^{2}=1$ for all $N$. Then $S_{N}$ is itself normally distributed with mean zero and variance one for all $N$ so that $P\left\{\left|S_{N}\right|>\varepsilon\right\}$ is a nonzero constant so the results of our theorems cannot hold no matter how fast $\rho_{N} \rightarrow 0$. The assumptions of Theorems 1c, 2c, 3, 4, and 5 all relate the rates at which $\rho_{N} \rightarrow 0$ and $\gamma_{N} \rightarrow 0$. The above argument shows that $\gamma_{N} \rightarrow 0$ is necessary because of considerations related to the Central Limit Theorem. Is it actually necessary that the rates at which $\rho_{N} \rightarrow 0$ and $\gamma_{N} \rightarrow 0$ be related, and if so what is the minimum restriction that need be put on the $\gamma_{N}$ 's?

It would be very nice to combine the assumptions on the moments with those on the coefficients in some way, and then to generalize this type of result so as to deal with probabilities of the form

$$
P\left\{\int f(t) d\left[X_{t}-E X_{t}\right] \geqq \varepsilon\right\}
$$


where $\left\{X_{t}\right\}$ is a continuous parameter process with independent increments. This sort of thing was done in a different situation in [7] and [8] but would appear to be harder in this case.

Also of interest are both exponential and algebraic rates of convergence for quadratic (and higher order) sums of independent random variables. At least in the quadratic case this convergence is of some practical interest. Unfortunately, obtaining results in these cases seems to be very hard and to require new techniques. The authors have been able to obtain only fragmentary results of this type.

\section{BiBLIOGRAPHY}

1. L. E. Baum and Melvin Katz, Convergence rates in the law of large numbers, Bull. Amer. Math. Soc. 69 (1963), 771-772.

2. - Convergence rates in the law of large numbers. II, Tech. Rep. No. 75, Dept. of Math. and Stat., Univ. of New Mexico, Albuquerque, 1964.

3. Y. S. Chow, Some convergence theorems for independent random variables, Ann. Math. Statist. 37 (1966), 1482-1493.

4. James Avery Davis, Convergence rates for the law of the iterated logarithm. Ann. Math. Statist. 39 (1968), 1479-1485.

5. - Convergence rates for probabilities of moderate deviations, Ann. Math. Statist. 39 (1968), 2016-2028.

6. W. E. Franck and D. L. Hanson, Some results giving rates of convergence in the law of large numbers for weighted sums of independent random variables, Trans. Amer. Math. Soc. 124 (1966), 347-359.

7. D. L. Hanson, Some results relating moment generating functions and convergence rates in the law of large numbers, Ann. Math. Statist. 38 (1967), 742-750.

8. D. L. Hanson and L. H. Koopmans, A probability bound for integrals with respect to stochastic processes with independent increments, Proc. Amer. Math. Soc. 16 (1965), 1173-1177.

9. C. C. Heyde, On almost sure convergence for sums of independent random variables, Res. Rep. No. 27, Dept. of Prob. and Stat., Univ. of Sheffield, 1967.

10. V. K. Rohatgi, On convergence rates in the law of large numbers for weighted sums of independent random variables, Res. Rep. No. 26, Dept. of Prob. and Stat., Univ. of Sheffield, 1967.

11. William Stout, Some results on the complete and almost sure convergence of linear combinations of independent random variables and martingale differences, Ann. Math. Statist. 39 (1968), 1549-1562.

\section{UNIVERSITY OF MISSOURI,} Columbia, Missouri 